\title{
Contribuição dos arranjos domiciliares para o suprimento de demandas assistenciais dos idosos com comprometimento funcional em São Paulo, Brasil
}

\author{
Yeda Aparecida de Oliveira Duarte, ${ }^{1}$ Maria Lúcia Lebrão \\ e Fernão Dias de Lima²
}

Como citar

Duarte YAO, Lebrão, ML, Lima FD. Contribuição dos arranjos domiciliares para o suprimento de demandas assistenciais dos idosos com comprometimento funcional em São Paulo, Brasil. Rev Panam Salud Publica. 2005;17(5/6):370-8.

RESUMO Objetivo. Apresentar os resultados do Projeto Saúde, Bem-Estar e Envelhecimento na América Latina e Caribe ( $S A B E$ ) no que tange ao desempenho funcional, às demandas assistenciais e aos arranjos familiares dos idosos do Município de São Paulo, Brasil.

Métodos. Em 2000, foram entrevistados 2143 indivíduos com 60 anos ou mais, utilizandose o questionário padronizado do SABE. A amostra foi obtida em dois estágios, utilizando-se setores censitários com reposição e com probabilidade proporcional à população. A complementação da amostra de pessoas de 75 anos ou mais foi realizada através da localização de moradias próximas aos setores selecionados. Os dados finais foram ponderados, de forma a serem expandidos. O desempenho funcional foi medido pela capacidade dos idosos de executar atividades básicas de vida diária ( $A B V D$, atravessar um cômodo da casa, comer, deitar-se e levantar da cama, usar o vaso sanitário, vestir-se e despir-se e tomar banho) e atividades instrumentais de vida diária (AIVD, comprar e preparar alimentos, realizar tarefas domésticas leves e pesadas, ir a outros lugares sozinho, usar o telefone, tomar os próprios medicamentos e manejar dinheiro), sendo a limitação funcional (demanda assistencial) definida como a necessidade de ajuda para executar pelo menos uma dessas atividades. Os 66 tipos de arranjos domiciliares identificados foram agrupados em sete categorias, de acordo com a presença ou não de co-residentes e o grau de parentesco entre o idoso e os co-residentes.

Resultados. Dos entrevistados, 19,2\% apresentaram limitações funcionais nas atividades básicas e 26,5\% nas atividades instrumentais, sendo a maior proporção entre mulheres e na velhice avançada. A proporção de idosos com limitações que recebeu ajuda variou, nas atividades básicas, de 25,6\% (para deitar-se e levantar da cama) a 70,5\% (para comer), e nas instrumentais, de 79,7\% (para a execução de tarefas domésticas leves) a 97,8\% (para comprar alimentos). Quanto aos arranjos familiares, a maioria dos idosos morava com o cônjuge e os filhos ou apenas com os filhos $(32,1 \%)$ ou somente com o cônjuge (20,0\%). O arranjo domiciliar em que a maior proporção de idosos com limitação funcional recebia ajuda $(56,4 \%)$ foi aquele no qual o idoso residia também com não-familiares.

Universidade de São Paulo (USP), Escola de Enfermagem. Enviar correspondência para esta autora no seguinte endereço: Escola de Enfermagem da
USP (EEUSP), Avenida Dr. Enéas de Carvalho Aguiar $419,3^{\circ}$ andar, sala 318, CEP 05403-000, São
Paulo, SP, Brasil. E-mail: yedaenf@usp.br e mllebr@ usp.br

2 USP, Faculdade de Saúde Pública. 
Conclusão. Os idosos com limitações funcionais não estão sendo assistidos suficientemente pela familia ou co-residentes, e as politicas públicas não suprem esse déficit assistencial. Dado o acelerado processo de envelhecimento da população, o sistema de assistência corre o risco de se tornar não só insuficiente, mas caótico.

Palavras-chave Atividades cotidianas, envelhecimento, relações familiares.

As mudanças demográficas decorrentes do processo de envelhecimento populacional, fenômeno que vem ocorrendo em alguns países latinoamericanos, dentre eles o Brasil, com espantosa velocidade, são acompanhadas por mudanças epidemiológicas que, por sua vez, geram a urgente necessidade de reorganizar a atenção à saúde do idoso. Isso se deve, em primeiro lugar, ao aumento na incidência de doenças crônico-degenerativas resultante do processo de envelhecimento populacional. Muitas vezes, essas doenças são acompanhadas por seqüelas limitantes de um desempenho funcional ótimo, o que pode gerar uma situação de dependência do idoso e ocasionar diferenciadas demandas assistenciais. É o grau de dependência que determinará quais tipos de cuidados serão necessários, e como e por quem os mesmos deverão ser realizados (1). Em segundo lugar, as sucessivas modificações estruturais observadas nas famílias, que se tornam cada vez mais nucleares e enfrentam expressivas modificações nos papéis desempenhados por seus membros, dificultam a participação da família na assistência ao idoso, o que pode contribuir para uma carência assistencial dos idosos mais incapacitados (2).

Entre janeiro de 2000 e março de 2001, a Organização Pan-Americana da Saúde (OPAS) coordenou, em sete países da América Latina e Caribe, o Projeto Saúde, Bem-Estar e Envelhecimento na América Latina e Caribe (SABE), cujo propósito foi realizar uma coleta sistemática de informações sobre as condições de vida dos idosos. No Brasil, o SABE foi realizado no $\mathrm{Mu}$ nicípio de São Paulo (3).

Os objetivos do presente trabalho foram apresentar os resultados do SABE no que tange ao desempenho funcional e aos arranjos familiares dos idosos do Município de São Paulo e analisar de que forma os arranjos domiciliares contribuem para suprir as demandas assistenciais dos idosos com comprometimento funcional.

\section{MATERIAIS E MÉTODOS}

O questionário SABE (4) foi composto de 11 seções que abrangem vários aspectos da vida do idoso, tais como dados pessoais, avaliação cognitiva, estado de saúde, estado funcional, medicamentos, uso e acesso aos serviços de saúde, rede de apoio familiar e social, história laboral e fontes de renda, características da moradia, antropometria, flexibilidade e mobilidade. $\mathrm{O}$ questionário foi aplicado a 2 143 pessoas de 60 anos ou mais residentes no Município de São Paulo.

Essa amostra foi composta por dois segmentos: o primeiro, resultante de sorteio, correspondeu à amostra probabilística formada por 1568 entrevistas. O segundo, formado por 575 residentes nos distritos em que se realizaram as entrevistas anteriores, correspondeu ao acréscimo efetuado para compensar a mortalidade na população de maiores de 75 anos e completar o número desejado de entrevistas nessa faixa etária.

Para o sorteio dos domicílios, usou-se o método de amostragem por conglomerados em dois estágios, sob o critério de partilha proporcional ao tamanho (5). Um cadastro permanente de 72 setores censitários, existente no Departamento de Epidemiologia da Faculdade de Saúde Pública da Universidade de São Paulo (USP), foi considerado como sendo a amostra de primeiro estágio. Essa amostra se originou do cadastro da Pesquisa Nacional por Amostra de
Domicílios (PNAD) de 1995, composto por 263 setores censitários sorteados sob o critério de probabilidade proporcional ao número de domicílios. O número mínimo de domicílios sorteados no segundo estágio foi aproximado para 90. A complementação da amostra de pessoas de 75 anos ou mais foi realizada através da localização de moradias próximas aos setores selecionados ou, no máximo, dentro dos limites dos distritos aos quais pertenciam os setores sorteados.

Cada questionário teve um peso calculado de acordo com o setor censitário de que fazia parte. Para os questionários realizados com indivíduos nos domicílios que foram selecionados, porém não sorteados (faixa etária de 75 anos e mais), o cálculo do peso foi realizado de acordo com a relação da população de idosos de ambos os sexos nessa faixa etária residentes no Município de São Paulo em 1998, e com o número de idosos nessa mesma faixa encontrado na amostra final do estudo (5).

Com base nas perguntas sobre estado funcional, foi feita a análise do desempenho funcional, observando-se dois pontos principais: se o idoso sentia dificuldade no desempenho de pelo menos uma atividade de vida diária (AVD), caracterizando o comprometimento funcional (ou a demanda assistencial); e, em caso de resposta positiva, se ele recebia ajuda para desempenhar essa atividade.

As AVD foram separadas em atividades básicas (ABVD, atravessar um cômodo da casa, comer, deitar-se e levantar da cama, usar o vaso sanitário, vestir-se e despir-se e tomar banho) e instrumentais (AIVD, comprar e preparar alimentos, realizar tarefas domésticas leves e pesadas, ir a outros lugares sozinho, usar o telefone, tomar 
os próprios medicamentos e manejar dinheiro), em virtude de cada conjunto de atividades gerar demandas assistenciais muito diferenciadas. $\mathrm{O}$ auxílio recebido dos co-residentes, conforme relato dos idosos que apresentavam comprometimento funcional, foi analisado de forma a investigar a existência de desequilíbrio entre a dificuldade na realização de ABVD e AIVD e o auxílio efetivamente recebido ou percebido pelo idoso.

Para tanto, a rede de suporte domiciliar dos idosos foi inicialmente identificada a partir de uma relação descritiva das várias combinações de co-residentes. Dado o número elevado de arranjos domiciliares identificados, foi preciso agrupar essas combinações para possibilitar uma análise mais adequada da relação entre as demandas referidas e a assistência recebida, enfoque principal deste estudo. Os arranjos domiciliares foram agrupados em sete categorias: o idoso mora sozinho (grupo A); mora somente com o cônjuge (grupo B); mora com o cônjuge e os filhos, ou mora com o cônjuge, os filhos e o genro ou a nora, ou mora somente com os filhos (grupo C); arranjos trigeracionais, ou seja, o idoso mora com filhos e netos (grupo D); arranjos intrageracionais, ou seja, o idoso mora somente com outros idosos além do cônjuge (grupo E); o idoso mora em arranjos intrageracionais ou somente com os netos (sem os filhos) (grupo F); e, finalmente, o idoso mora com não-familiares (grupo G).

$O$ teste do qui-quadrado $\left(\chi^{2}\right)$ foi usado para verificar as seguintes associações: desempenho funcional versus faixa etária (60 a 74 anos, 75 anos e mais) e sexo; e demandas assistenciais (comprometimento funcional) versus tipo de arranjo familiar. O nível de significância foi fixado em $0,01(\alpha=1 \%)$.

\section{RESULTADOS}

\section{Atividades básicas de vida diária}

A maioria dos idosos $(80,7 \%)$ não apresentou limitações na execução das ABVD. Dos 19,2\% que apresentaram limitações funcionais, $70,5 \%$ as apresentaram em uma ou duas atividades, e 29\% em três ou mais. Quando essa análise foi estratificada por faixa etária e sexo, observou-se uma piora de desempenho com o avançar da idade (um aumento no comprometimento funcional em três ou mais atividades), sendo tal situação mais acentuada entre os homens (tabela 1). Dentre as ABVD, vestir-se, deitar-se e levantar da cama e tomar banho foram as que apresentaram os maiores índices de dificuldade. Em todas as atividades, a proporção de mulheres com dificuldade foi maior do que a proporção de homens, com exceção da alimentação (tabela 2).

Quando os idosos com dificuldade foram perguntados se recebiam ajuda para desempenhar as ABVD, observouse um desequilíbrio entre a demanda assistencial e o recebimento de auxílio (tabela 3). Em geral e, mais especificamente, na faixa de 60 a 74 anos, as mulheres recebiam menos auxílio do que os homens, a não ser para comer. Todas as diferenças encontradas foram estatisticamente significativas, com valores de $P$ que variaram de 0,0000 a 0,0010 .

\section{Atividades instrumentais de vida diária}

Em relação às AIVD, 73,4\% dos idosos pesquisados não apresentavam dificuldades. Observou-se que as dificuldades apresentadas por $26,5 \%$ deles foram mais acentuadas entre as mulheres e pioraram expressivamente na velhice avançada em ambos os sexos (tabela 1). "Ir a outros lugares sozinho", que neste estudo relacionou-se diretamente ao uso de transportes coletivos, mostrou-se a atividade mais comprometida em ambos os sexos, sendo muito mais limitante para as mulheres do que para os homens (tabela 2). "Fazer compras de alimentos", na terceira posição em freqüência,

TABELA 1. Distribuição dos idosos segundo o sexo, o grupo etário e o número de atividades de vida diária executadas com dificuldade, São Paulo (SP), Brasil, 2000

\begin{tabular}{|c|c|c|c|c|c|c|c|}
\hline \multirow[b]{2}{*}{$\begin{array}{l}\text { Número de atividades } \\
\text { com dificuldade }\end{array}$} & \multicolumn{3}{|c|}{ Feminino (\%) } & \multicolumn{3}{|c|}{ Masculino (\%) } & \multirow[b]{2}{*}{$\begin{array}{c}\text { Total } \\
(\%)\end{array}$} \\
\hline & $\begin{array}{c}60 \text { a } 74 \\
\text { anos }\end{array}$ & $\begin{array}{l}75 \text { anos } \\
\text { ou mais }\end{array}$ & Total & $\begin{array}{c}60 \text { a } 74 \\
\text { anos }\end{array}$ & $\begin{array}{l}75 \text { anos } \\
\text { ou mais }\end{array}$ & Total & \\
\hline \multicolumn{8}{|l|}{ Atividades básicas ${ }^{a}$} \\
\hline 1 a 2 & 78,8 & 60,3 & 71,8 & 74,8 & 54,0 & 67,6 & 70,5 \\
\hline 3 ou mais & 20,5 & 38,7 & 27,4 & 25,2 & 46,0 & 32,4 & 29,0 \\
\hline Não respondeu & 0,7 & 1,0 & 0,8 & - & - & - & 0,5 \\
\hline Total & 100,0 & 100,0 & 100,0 & 100,0 & 100,0 & 100,0 & 100,0 \\
\hline \multicolumn{8}{|l|}{ Atividades instrumentais ${ }^{b}$} \\
\hline 1 a 2 & 69,3 & 53,3 & 62,3 & 49,5 & 46,1 & 48,1 & 58,2 \\
\hline 3 ou mais & 30,2 & 45,9 & 37,0 & 50,5 & 53,9 & 51,9 & 41,3 \\
\hline Não respondeu & 0,5 & 0,8 & 0,7 & - & - & - & 0,5 \\
\hline Total & 100,0 & 100,0 & 100,0 & 100,0 & 100,0 & 100,0 & 100,0 \\
\hline
\end{tabular}

\footnotetext{
a Atravessar um cômodo da casa, comer, deitar-se e levantar da cama, usar o vaso sanitário, vestir-se e despir-se e tomar banho.

${ }^{\mathrm{b}}$ Comprar e preparar alimentos, realizar tarefas domésticas leves e pesadas, ir a outros lugares sozinho, usar o telefone, tomar os próprios medicamentos e manejar dinheiro.
} 
TABELA 2. Distribuição dos idosos segundo o sexo, o grupo etário e o tipo de atividade de vida diária executada com dificuldade, São Paulo (SP), Brasil, 2000

\begin{tabular}{|c|c|c|c|c|c|c|c|}
\hline \multirow[b]{2}{*}{ Atividades de vida diária } & \multicolumn{3}{|c|}{ Feminino (\%) } & \multicolumn{3}{|c|}{ Masculino (\%) } & \multirow[b]{2}{*}{$\begin{array}{c}\text { Total } \\
(\%)\end{array}$} \\
\hline & $\begin{array}{c}60 \text { a } 74 \\
\text { anos }\end{array}$ & $\begin{array}{l}75 \text { anos } \\
\text { ou mais }\end{array}$ & Total & $\begin{array}{c}60 \text { a } 74 \\
\text { anos }\end{array}$ & $\begin{array}{l}75 \text { anos } \\
\text { ou mais }\end{array}$ & Total & \\
\hline \multicolumn{8}{|l|}{ Vestir-se (inclusive calçar } \\
\hline sapatos e meias) & 12,8 & 22,7 & 15,2 & 8,2 & 23,5 & 11,2 & 13,5 \\
\hline \multicolumn{8}{|l|}{ Mobilização (deitar-se e } \\
\hline cômodo caminhando) & 3,0 & 15,7 & 6,0 & 3,4 & 10,2 & 4,7 & 5,5 \\
\hline Usar o vaso sanitário & 3,9 & 11,2 & 5,6 & 2,4 & 9,8 & 3,8 & 4,9 \\
\hline Comer & 1,7 & 7,7 & 3,1 & 3,0 & 9,6 & 4,3 & 3,6 \\
\hline \multicolumn{8}{|l|}{ Instrumentais } \\
\hline Ir a outros lugares sozinho & 16,2 & 42,7 & 22,6 & 6,3 & 28,4 & 10,6 & 17,6 \\
\hline Executar tarefas domésticas leves & 4,6 & 8,1 & 5,4 & 2,1 & 5,5 & 2,8 & 4,3 \\
\hline Preparar refeições & 2,2 & 8,2 & 3,6 & 2,1 & 8,2 & 3,3 & 3,5 \\
\hline
\end{tabular}

TABELA 3. Distribuição dos idosos que recebiam ajuda para executar atividades de vida diária segundo o sexo, o grupo etário e o tipo de atividade, São Paulo (SP), Brasil, 2000

\begin{tabular}{|c|c|c|c|c|c|c|c|}
\hline \multirow[b]{2}{*}{ Atividades de vida diária } & \multicolumn{3}{|c|}{ Feminino (\%) } & \multicolumn{3}{|c|}{ Masculino (\%) } & \multirow[b]{2}{*}{$\begin{array}{l}\text { Total } \\
(\%)\end{array}$} \\
\hline & $\begin{array}{l}60 \text { a } 74 \\
\text { anos }\end{array}$ & $\begin{array}{l}75 \text { anos } \\
\text { ou mais }\end{array}$ & Total & $\begin{array}{l}60 \text { a } 74 \\
\text { anos }\end{array}$ & $\begin{array}{l}75 \text { anos } \\
\text { ou mais }\end{array}$ & Total & \\
\hline \multicolumn{8}{|l|}{ Básicas } \\
\hline Comer & 68,6 & 74,6 & 72,1 & 69,1 & 68,5 & 68,8 & 70,5 \\
\hline Tomar banho & 48,4 & 75,8 & 62,1 & 74,5 & 64,5 & 70,2 & 65,6 \\
\hline Locomoção (atravessar um & & & & & 10 & & \\
\hline $\begin{array}{l}\text { Mobilização (deitar-se e } \\
\text { levantar da cama) }\end{array}$ & 14,5 & 37,3 & 22,2 & 34,3 & 35,7 & 34,7 & 25,6 \\
\hline \multicolumn{8}{|l|}{ Instrumentais } \\
\hline Comprar alimentos & 99,7 & 95,6 & 97,8 & 97,7 & 98,3 & 97,9 & 97,8 \\
\hline Manejar dinheiro & 95,8 & 96,6 & 96,3 & 93,3 & 95,2 & 94,1 & 95,4 \\
\hline Ir a outros lugares sozinho & 86,5 & 96,6 & 91,4 & 100,0 & 89,4 & 94,8 & 92,2 \\
\hline Executar tarefas domésticas leves & 61,2 & 82,5 & 71,5 & 100,0 & 96,9 & 98,7 & 79,7 \\
\hline
\end{tabular}

manteve a mesma relação descrita acima. Tais resultados revelam uma tendência à redução das atividades externas ao domić́lio. Outro fato a res- saltar é que 8,5\% dos idosos — cerca de 85000 pessoas no Município de São Paulo- referiram dificuldades para tomar os próprios medicamentos, fato esse que pode comprometer o tratamento desses indivíduos. Quando confrontadas as atividades instrumentais entre os sexos, foram encontradas 
TABELA 4. Distribuição dos idosos segundo o sexo e o tipo de arranjo familiar e proporção dos idosos com dificuldade em executar atividades básicas de vida diária que recebiam ajuda segundo o número de atividades e o tipo de arranjo familiar, São Paulo (SP), Brasil, 2000

\begin{tabular}{|c|c|c|c|c|c|c|c|c|c|}
\hline \multirow[b]{2}{*}{ Arranjo familiarb } & \multicolumn{4}{|c|}{ Sexo (\%) } & \multirow[b]{2}{*}{$\begin{array}{l}\text { Total } \\
\text { (posto) }\end{array}$} & \multicolumn{3}{|c|}{$\begin{array}{l}\text { Ajuda recebida por dificuldades apresentadas nas } \\
\text { atividades básicas de vida diária }{ }^{\mathrm{a}}\end{array}$} & \multirow[b]{2}{*}{ Total } \\
\hline & \multicolumn{2}{|c|}{$\begin{array}{l}\text { Feminino } \\
\text { (posto) }\end{array}$} & \multicolumn{2}{|c|}{$\begin{array}{l}\text { Masculino } \\
\text { (posto) }\end{array}$} & & \multirow{2}{*}{$\begin{array}{c}\begin{array}{c}\text { Não recebe } \\
\text { ajuda }\end{array} \\
83,6\end{array}$} & \multirow{2}{*}{$\begin{array}{c}\begin{array}{c}1 \text { ou } 2 \\
\text { atividades }\end{array} \\
14,5\end{array}$} & \multirow{2}{*}{$\begin{array}{c}\begin{array}{l}3 \text { ou mais } \\
\text { atividades }\end{array} \\
1,9\end{array}$} & \\
\hline A & 17,1 & $\left(3^{\circ}\right)$ & 7,5 & $\left(4^{\circ}\right)$ & $13,1 \quad\left(4^{\circ}\right)$ & & & & 100,0 \\
\hline B & 15,6 & $\left(4^{\circ}\right)$ & 26,2 & $\left(2^{\circ}\right)$ & $20,0 \quad\left(2^{\circ}\right)$ & 64,0 & 17,8 & 18,2 & 100,0 \\
\hline C & 29,8 & $\left(1^{\circ}\right)$ & 35,4 & $\left(1^{\circ}\right)$ & $32,1 \quad\left(1^{\circ}\right)$ & 59,4 & 29,2 & 11,4 & 100,0 \\
\hline G & 6,8 & $\left(5^{\circ}\right)$ & 7,0 & $\left(6^{\circ}\right)$ & $6,9 \quad\left(5^{\circ}\right)$ & 43,6 & 29,8 & 26,6 & 100,0 \\
\hline Total & $10 c$ & & 10 & & 100,0 & 60,6 & 24,1 & 15,3 & 100,0 \\
\hline
\end{tabular}

a Atravessar um cômodo da casa, comer, deitar-se e levantar da cama, usar o vaso sanitário, vestir-se e despir-se e tomar banho.

b Grupo A: o idoso mora sozinho; grupo B: mora somente com o cônjuge; grupo C: mora com o cônjuge e os filhos, ou com o cônjuge, os filhos e o genro ou a nora, ou somente com os fiIhos; grupo D: arranjos trigeracionais (idoso, filhos e netos); grupo E: arranjos intrageracionais (idoso mora somente com outros idosos além do cônjuge); grupo F: mora em arranjos intrageracionais ou somente com os netos (sem os filhos); grupo G: mora com não-familiares.

diferenças estatísticas significativas ( $P$ variando de 0,0000 a 0,1763 ), exceto para "tomar medicamentos" e "preparar refeições" ( $P=0,0576$ e 0,1763, respectivamente).

\section{Arranjos domiciliares}

Sessenta e seis tipos de arranjos domiciliares foram identificados na relação descritiva das várias combinações de co-residentes entre os idosos no Município de São Paulo. Levando em consideração os sete grupos de arranjos domiciliares estabelecidos neste estudo, observou-se que o grupo mais freqüente foi o dos idosos que residiam com o cônjuge ou os filhos (sem os netos); em segundo lugar estava o grupo dos que residiam somente com o cônjuge; em terceiro, o grupo dos arranjos trigeracionais; e em quarto, o grupo dos idosos que moravam sozinhos.

A tabela 4 mostra a distribuição dos idosos de acordo com o sexo e o grupo de arranjos domiciliares. A proporção de mulheres morando sozinhas foi maior do que a de homens, que residiam mais com o cônjuge e os filhos ou somente com o cônjuge. As idosas, por sua vez, foram mais acolhidas em arranjos bi- ou trigeracionais. Comparando-se os arranjos domiciliares por sexo, foram encontradas di- ferenças estatísticas significativas em todos os arranjos, com $P$ variando de 0,0000 a 0,0004 .

A tabela 4 mostra ainda a porcentagem de idosos com dificuldade em executar ABVD que recebiam auxílio para a execução dessas atividades. Verificou-se que 60,6\% referiram não receber qualquer tipo de auxílio. Os arranjos que mais supriam as demandas assistenciais relacionaram-se aos arranjos trigeracionais e àqueles que contavam com a presença de nãofamiliares, possivelmente cuidadores.

\section{DISCUSSÃO}

A funcionalidade apresentada pelos idosos, determinada a partir da avaliação do desempenho de AVD em associação à assistência recebida pelos idosos para a execução dessas tarefas em cada grupo de arranjos domiciliares, é um dos alicerces da organização das políticas públicas relacionadas à melhoria da qualidade assistencial destinada a esse grupo etário. Este estudo buscou traçar um panorama dessa realidade.

As ABVD estão relacionadas ao desempenho do cuidado pessoal. $\mathrm{O}$ seu comprometimento implica, freqüentemente, a necessidade de auxílio direto por parte de outras pessoas, tornandoas, assim, onerosas e complexas (2).
Para o Município de São Paulo, a proporção de idosos com esse tipo de comprometimento funcional $(19,2 \%)$ representava, aproximadamente, 200000 pessoas com limitações relacionadas à sobrevivência e a uma boa qualidade de vida. Desses, 29\% relataram comprometimento em três ou mais atividades, indicando, nesse caso, alta dependência de cuidados. Tal informação vai ao encontro da necessidade, cada vez mais presente, de se identificar as pessoas com incapacidades importantes e que ficam à margem do sistema de saúde, já que apresentam um tipo de incapacidade que não é contemplado pelo sistema e, portanto, não são por ele conhecidas.

$\mathrm{Na}$ análise por sexo, verificou-se que as mulheres apresentaram um pior desempenho em todas as atividades (exceto para comer). Esse dado é reforçado pelo estudo de Santos (6), que, utilizando a mesma amostra populacional do presente estudo, analisou a sobrevida de idosos sem incapacidade e constatou que, embora o sexo masculino tenha menor esperança de vida, a proporção dos anos a serem vividos sem incapacidades é maior do que no sexo feminino. Os mais idosos também apresentaram dificuldade em um número maior de atividades, o que implica uma situação de maior vulnerabilidade e, conseqüentemente, de maior risco. 
Verificou-se que a ajuda recebida, referida pelos idosos com dificuldades, em nenhuma das situações aproximou-se de $100 \%$. Apenas na atividade relacionada à alimentação a proporção de auxílio recebido foi mais elevada. Isso se dá, possivelmente, por estar essa atividade ligada diretamente à questão da sobrevivência do idoso. Ainda, o não-atendimento de tal necessidade poderia ser interpretado como maus-tratos, com desdobramentos legais. Para as outras atividades, relacionadas a bem-estar e conforto, o auxílio recebido mostrouse muito aquém das demandas referidas, comprometendo, dessa forma, a qualidade de vida dos idosos. A análise estratificada por sexo mostrou que os homens recebem maior auxílio. Isso sugere uma provável influência do estado conjugal, já que há mais mulheres sem companheiro e, entre os homens, é bem conhecido o suporte que as mulheres oferecem.

A avaliação do desempenho funcional dos idosos nas AIVD - relacionadas menos com o cuidado direto e mais com a participação efetiva do idoso na comunidade e com a distribuição de tarefas entre os membros familiares (responsabilizar-se por fazer compras ou auxiliar nas tarefas domésticas)_mostrou comprometimento em um número maior de idosos do que nas ABVD (26,5\%). Esses dados reforçam a já conhecida relação hierárquica de perda de habilidades funcionais que ocorre no envelhecimento, e que se direciona, normalmente, das AIVD para as ABVD (7). Tais idosos necessitam, portanto, do auxílio de outras pessoas que atuem como elo de ligação com o entorno social. Esse tipo de limitação tende a contribuir para o maior afastamento do idoso do convívio social e, conseqüentemente, para o isolamento. Considerando os estudos que relacionam os quadros de isolamento com os quadros depressivos, tais achados merecem atenção diferenciada.

A análise das AIVD revelou ainda importantes influências culturais. Os homens apresentaram uma tendência a não executar certas atividades, como, por exemplo, preparar refeições ou fazer tarefas domésticas, fossem elas pesadas ou leves. Isso reforça a necessidade de um maior detalhamento desse tipo de análise, de forma a diferenciar a dificuldade funcional da influência cultural. A não-observância de tais questões pode gerar falsos resultados no que tange às necessidades dos idosos, dificultando, dessa forma, a organização e a administração adequada de suas reais demandas.

Os resultados encontrados neste estudo mostraram uma tendência à redução das atividades externas ao domicílio, dada a dificuldade em sair do mesmo, tanto para atividades mais essenciais quanto para outras nem tão essenciais. Em última análise, isso gera uma diminuição progressiva dos contatos externos e do círculo de relacionamentos e, portanto, reduz a rede de apoio social e ocasiona maior dependência de outras pessoas para promover a integração do idoso à sociedade; de certa forma, essa situação pode levar a uma sobrecarga familiar (2).

Outro resultado que preocupa foi a dificuldade referida para a autoadministração de medicamentos. A maioria dos idosos que relataram tal dificuldade é portadora de doenças crônicas que exigem o uso de medicamentos contínuos. Nesses casos, deixar de tomar os medicamentos pode representar uma pior evolução das doenças preexistentes, aumentando a possibilidade de ocorrência de seqüelas ainda mais limitantes (8).

A ajuda recebida nas AIVD surpreendentemente superou, em muito, a ajuda recebida nas ABVD, e, excetuando-se o manejo de dinheiro, os homens receberam mais auxílio do que as mulheres, fato esse que se acentuou com o avançar da idade. É, ainda, interessante observar que, apenas para os homens, em algumas dessas atividades, o percentual de ajuda atingiu $100 \%$. Isso possivelmente ocorre em virtude de a ajuda demandada nessas atividades exigir mais uma reorganização familiar do que um auxílio direto por parte de outras pessoas. Os menores percentuais de auxílio observados referiram-se às idosas mais jovens em relação às tarefas domésticas leves, seguidas pelas pesadas, reforçando o padrão cultural ainda predominante na sociedade brasileira. Embora o envelhecimento e o declínio no desempenho funcional atinjam ambos os sexos, aparentemente a sobrecarga feminina perdura por maior tempo, pois é provável que a mulher, pela sobrevida maior, continue a cuidar de seu cônjuge e de outros familiares (9).

A questão preponderante em relação à velhice atualmente está, portanto, relacionada ao equilíbrio entre as necessidades e as exigências mínimas dos idosos e a capacidade assistencial disponível para atendê-las, em nível familiar ou coletivo, pois, nessa fase da vida, tais aspectos adquirem características especiais (10). Até o momento, em nenhuma sociedade estudada, mais de $10 \%$ dos idosos são institucionalizados (11). Isso torna fundamental a compreensão dos contextos familiares, pois eles representam a grande fonte de auxílio com que os idosos, em geral, poderão contar. Espera-se da família o cumprimento do que se julga ser seu papel fundamental, ou seja, assegurar o bem-estar afetivo e material e absorver o impacto das tensões, adaptando-se às diferentes demandas e mantendo um equilíbrio dinâmico em sua estrutura $(2,12)$.

Os arranjos domiciliares podem ser classificados de formas diversas. Um estudo retrospectivo sobre a classificação dos arranjos domiciliares e nucleares no Brasil optou por uma divisão genérica, considerando como arranjos familiares os nucleares, os estendidos e os complexos, e como arranjos não-familiares aqueles individuais e outros (13). Há autores que destacam a presença ou não de filhos, pois esses representam, ainda hoje, o maior potencial de auxílio aos idosos, agindo, dessa forma, como fator de proteção (14). Foi objetivo deste estudo, no entanto, apresentar os arranjos aparentemente mais vulneráveis, de forma a propiciar uma reorganização assistencial mais efetiva. O agrupamento ora proposto levou em conta basicamente as relações intra- (entre idosos) e intergeracionais (15).

A condição de residir sozinho (grupo A) ocorreu com maior freqüên- 
cia entre as mulheres, possivelmente dada a maior probabilidade de casar novamente observada entre os homens. No caso dos arranjos que envolviam apenas o casal de idosos (grupo B), as demandas são, geralmente, parcialmente atendidas e tendem a sobrecarregar a mulher, na maioria das vezes também idosa ou muito próxima dessa faixa etária, podendo ter seu potencial de auxílio mais limitado. A suplementação de auxílio por parte dos filhos ou de outros familiares tende a surgir apenas mais tardiamente. Estudos mostram que as esposas idosas assumem freqüentemente a função de cuidadoras, embora nem sempre estejam fisicamente aptas para tal $(2,9,16$, 17). Nos arranjos em que convivem os idosos e seus filhos (grupo C), a ajuda geralmente provém de uma filha, ou, quando necessário, dos dois idosos. Os arranjos trigeracionais (grupo D) diferem do grupo anterior pela introdução da terceira geração, o que pode levar a uma hierarquização na distribuição de recursos materiais para o atendimento às demandas da própria família. Nesse caso, a tendência é que as demandas dos idosos sejam preteridas em benefício dos mais jovens. No caso dos arranjos intrageracionais (grupo E), envolvendo essencialmente idosos, as demandas tendem a estar acumuladas, e o potencial de ajuda tende a ser mais limitado. No arranjo que inclui avós e netos, sem filhos co-residentes (grupo F), a ajuda tende a ser direcionada mais dos idosos para os netos, enquanto o sentido contrário tende a ser muito precário. Estudos realizados por pesquisadores espanhóis colocam os avós como âncoras situacionais, pois são capazes de transmitir às gerações mais jovens, além da história social geral, a da própria família, proporcionando-lhes, assim, referências sociais mais sólidas $(18,19)$. Por último encontram-se os arranjos onde existe um auxílio extrafamiliar presente (grupo G), remunerado ou não. Esse arranjo ocupou o último lugar em freqüência para ambos os sexos, demonstrando que a família ainda é o principal recurso disponível para atender às demandas dos idosos $(2,13,20)$.
$\mathrm{Na}$ maioria das cidades pesquisadas no SABE, com exceção de Havana (Cuba) e da Cidade do México (México), o principal arranjo observado foi o casal sem outras pessoas $\mathrm{CO}^{-}$ residentes. Um dado preocupante foi o contingente de idosos que moravam sozinhos, ocupando o segundo lugar em três das sete cidades (Buenos Aires, Bridgetown e Montevidéu). Em São Paulo (Brasil), Santiago (Chile) e Havana, esse arranjo ocupou o terceiro posto, ficando na quinta posição apenas na Cidade do México, onde se observa uma maior proporção de co-residentes.

Nos Estados Unidos, as famílias provêem de 80 a $90 \%$ dos cuidados aos idosos na comunidade. Numericamente, 2,7 milhões de filhos respondem pelos cuidados de seus pais idosos incapacitados. Observa-se, ainda, um aumento no número de idosos fragilizados com múltiplos problemas de saúde que, em muitas ocasiões, são cuidados por outros idosos (11). Com a saída dos mais jovens das casas, tende a diminuir o número de famílias que agregam três ou quatro gerações. Mudanças nos padrões de mortalidade, fecundidade e nupcialidade, e a presença, cada vez mais freqüente, de divórcios e famílias de estilo alternativo, também afetam o equilíbrio entre as necessidades dos indivíduos e a disponibilidade real de auxílio. Quando um cuidador está disponível, a carga sobre ele pode ser muito importante. Há que se ressaltar, ainda, que, na atual conjuntura socioeconômica, muitos filhos estão voltando a residir na casa dos pais, em decorrência de questões financeiras, muitas vezes acompanhados de suas novas famílias e com demandas diferenciadas. Esse fato pode, em última análise, interferir no potencial de ajuda disponível $(20,21)$.

Nos arranjos apresentados neste estudo, observa-se uma tendência masculina de residir com o cônjuge ou os filhos, ou somente com o cônjuge. Esse resultado mostra a existência de um maior potencial de ajuda para os homens do que para as mulheres. As idosas, por outro lado, foram mais acolhidas em arranjos bi- ou trigeracionais, neste último caso provavelmente pela contribuição que podem oferecer, por exemplo, para o cuidado dos netos.

Os resultados encontrados relacionados à ajuda fornecida dentro dos diferentes arranjos domiciliares são extremamente preocupantes. Verificouse que, entre os arranjos que incluíam a presença de familiares, os que mais supriram a demanda assistencial foram os que contavam com a presença de filhos, corroborando o encontrado na literatura $(14,20)$, onde a co-residência mostrou-se mais importante do que o número de filhos. Porém, mesmo nessas circunstâncias, apenas em torno de $50 \%$ dos indivíduos com dificuldade recebiam ajuda, o que está muito aquém do necessário. A ajuda mais expressiva foi observada em arranjos que contavam com a presença de nãofamiliares, que incluíam, provavelmente, cuidadores contratados. Nesses, 43,6\% dos idosos referiram não receber ajuda, o menor percentual encontrado no estudo. Os arranjos que contavam somente com idosos (intrageracionais ou somente cônjuge) não supriram adequadamente as demandas assistenciais dos mesmos, embora o arranjo onde o idoso residia somente com o cônjuge tenha representado o segundo tipo mais freqüente no estudo. $\mathrm{O}$ retrato ora observado mostra que as necessidades apresentadas pelos idosos estão sendo inadequadamente atendidas pelos arranjos domiciliares existentes. Tal fato torna-se ainda mais grave quando se observa que poucas são as medidas que vêm sendo tomadas no sentido de minimizar o sofrimento a que esses idosos e suas famílias acabam, muitas vezes, por ser submetidos.

Frente às transformações nas estruturas familiares, multiplicam-se os questionamentos quanto ao impacto causado pela presença de idosos com limitações importantes na dinâmica de funcionamento das famílias. Supõe-se que, em condições de disfuncionalidade das famílias, essas possam ter sua capacidade assistencial diminuída e, assim, não ser capazes de prover adequadamente o atendimento sistemático das necessidades dos idosos. A capacidade de identificar essas demandas de forma adequada pode estar 
prejudicada, assim como o reconhecimento de quais cuidados são necessários e de quem poderia, mais apropriadamente, fornecê-los (2).

\section{CONCLUSÕES}

Os resultados obtidos neste trabalho mostram que os idosos, em sua maioria, encontram-se com bom desempenho funcional e, conseqüentemente, com menores demandas assistenciais, residindo em arranjos familiares multi- geracionais. Contudo, os idosos que apresentam limitações funcionais não estão sendo assistidos adequadamente. Os arranjos domiciliares existentes não estão sendo capazes de atender, de forma global, às necessidades mais básicas apresentadas pelos idosos, tornando-os, assim, mais vulneráveis. Até o momento, não há no Brasil uma política para suprir tais deficiências. Dado o processo de envelhecimento da população, ainda mais acelerado na velhice avançada, o sistema de assistência corre o risco de passar, em breve, de insuficiente a caótico.

Agradecimentos. Os autores agradecem à Dra. Martha Peláez, pela coordenação competente e afetuosa do Projeto SABE e por, juntamente com o Prof. Alberto Palloni, tornar este estudo uma realidade; e à Professora Sabina L. D. Gotlieb, pela leitura atenta e pela análise estatística. O Projeto SABE foi financiado pela Fundação de Amparo à Pesquisa do Estado de São Paulo (FAPESP) (processo no 99/05125-7).

\section{REFERÊNCIAS}

1. Baltes MM, Silvenberg S. A dinâmica dependência-autonomia no curso de vida. Em: Neri AL, ed. Psicologia do envelhecimento: temas selecionados na perspectiva do curso de vida. Campinas: Papirus; 1995.(Coleção Viva Idade).

2. Duarte YAO. Família: rede de suporte ou fator estressor. A ótica de idosos e cuidadores familiares [tese de doutorado]. São Paulo: Escola de Enfermagem, Universidade de São Paulo; 2001.

3. Lebrão ML, Duarte YAO. O Projeto Sabe no Município de São Paulo: uma abordagem inicial. Brasília: Organização Pan-Americana da Saúde; 2003.

4. Questionário do Projeto Saúde, Bem-Estar e Envelhecimento na América Latina e Caribe (SABE). Disponível em: http:/www.fsp.usp. $\mathrm{br} /$ sabe . Acessado em fevereiro de 2005.

5. Silva NN. Aspectos metodológicos: processo de amostragem. Em: Lebrão ML, Duarte YAO, orgs. O projeto SABE no município de São Paulo: uma abordagem inicial. Brasília: Organização Pan-Americana da Saúde, Ministério da Saúde; 2003. Pp. 47-57.

6. Santos JLF. Análise de sobrevida sem incapacidade. Em: Lebrão ML, Duarte YAO, orgs. O projeto SABE no município de São Paulo: uma abordagem inicial. Brasília: Organização PanAmericana da Saúde, Ministério da Saúde; 2003. Pp. $167-81$.
7. Ramos LR, Perracini M, Rosa TEC, Kalache A. Significance and management of disability among urban elderly residents in Brazil. J Cross Cult Gerontol. 1993;8(4):313-23.

8. Secoli S, Duarte YAO. Medicamentos e a assistência domiciliar. Em: Duarte YAO, Diogo MJD, eds. Atendimento domiciliar: um enfoque gerontológico. São Paulo: Atheneu; 2000. Pp. 326-35.

9. Karsch UMS. Envelhecimento com dependência: revelando cuidadores. São Paulo: Pontifícia Universidade Católica de São Paulo; 1998.

10. Motlis J. La vejez y sus múltiples caras. TelAviv: Aurora; 1998.

11. Older Women's League. Administration on aging awards support for national caregiving information project. Washington, D.C.: OWL Press; 1992.

12. Pintos $\mathrm{CCH}$. A família e a terceira idade: orientações psicogerontológicas. São Paulo: Paulinas; 1997.

13. Medeiros M, Osório R. Arranjos domiciliares e arranjos nucleares no Brasil: classificação e evolução de 1977 a 1998. Brasília: Instituto de Pesquisa Econômica Aplicada; 2001.

14. Saad PM. Transferencias informales de apoyo de los adultos mayores en América Latina y el Caribe: estudio comparativo de encuestas SABE. Notas Poblacion. 2003;3(77):175-217.

15. Ebersole P, Hess P. Relationships and their significance. Em: Ebersole P, Hess P, eds. To- ward healthy aging: human needs and nursing response. $5^{\mathrm{a}}$ ed. St. Louis: Mosby; 1998. Pp. 606-39.

16. Schulz R, O'Brien AT, Bookwala J, Fleissner K. Psychiatric and physical morbidity of dementia caregiving: prevalence, correlates and causes. Gerontologist. 1995;35(6):771-9.

17. Nielsen J, Henderson C, Cox M, Williams S, Green P. Characteristics of caregivers and factors contributing to institutionalization. Geriatr Nurs. 1996;17(3):120-8.

18. Moragas RM. Gerontologia social: envelhecimento e qualidade de vida. São Paulo: Paulinas; 1997.

19. Moragas RM. Gerontología social. Barcelona: Herder; 1991.

20. Hays JC. Living arrangements and health status in later life: a review of recent literature. Public Health Nurs. 2002;199(2):136-51.

21. Chaimowicz F. Os idosos brasileiros no século XXI: demografia, saúde e sociedade. Belo Horizonte: Postgraduate; 1998.

Manuscrito recebido em 29 de junho de 2004. Aceito em versão revisada em 7 de janeiro de 2005 . 
ABSTRACT Objectives. To describe the functional performance, need for assistance, and living arrangements of elderly persons in the city of São Paulo, Brazil, as part of a project called Health, Well-being, and Aging in Latin America and the Caribbean (the "SABE project").

The contribution of living arrangements in the provision of care for elderly persons with functional impairments in São Paulo, Brazil
Methods. In 2000, 2143 individuals 60 years of age or older were interviewed, using the standardized SABE questionnaire. The sample was obtained in two stages, using census sectors with replacement and probability proportional to population. To have the desired number of respondents 75 or older, additional homes close to the selected census sectors were found, with weighting of the final results. The functional performance of the elderly persons was measured in terms of their ability to perform basic activities of daily living (walking across a room, eating, lying down on a bed and getting up from it, using the bathroom, dressing and undressing, and bathing) and to carry out instrumental activities of daily living (buying and preparing food, performing light and heavy household chores, using the telephone, taking medications, and handling money). Functional impairment was defined as the need for assistance in performing at least one of the basic or instrumental activities. There were 66 types of living arrangements (household composition) identified and grouped into seven categories, according to with whom an elderly person lived and whether the other residents of the household were relatives or not.

Results. Of the individuals interviewed, $19.2 \%$ presented a functional impairment in basic activities and $26.5 \%$ in instrumental activities. The impairments were more common among women and among persons 75 or older. The proportion of elderly persons with an impairment who received help with basic activities ranged from $25.6 \%$ (lying down on the bed and getting up) to $70.5 \%$ (eating). With instrumental activities, the proportion receiving assistance ranged from $79.7 \%$ (light domestic chores) to $97.8 \%$ (buying food). With respect to living arrangements, the single largest group $(32.1 \%)$ of elderly persons lived with the spouse and children or with just the children; the next largest group (20.0\%) lived with just the spouse. The living arrangement in which the highest proportion $(56.4 \%)$ of elderly persons with functional impairments received help was living with nonrelatives.

Conclusions. The elderly persons with functional impairments in São Paulo are not receiving enough assistance from their family members or other persons with whom they live. Further, there are no public policies in Brazil to fill this gap. Taking into account the country's aging population, the health care system in Brazil is at risk of becoming not only inadequate but chaotic.

Keywords Activities of daily living, aging, family relations. 